Revta brasil. Bot., São Paulo, V.24, n.3, p.283-288, set. 2001

\title{
Sistemas reprodutivos de espécies de Melastomataceae da Serra do Japi, Jundiaí, São Paulo, Brasil
}

\author{
RENATO GOLDENBERG ${ }^{1,3}$ e ISABELA GALARDA VARASSIN ${ }^{2}$
}

(recebido: 13 de dezembro de 2000; aceito: 30 de maio de 2001)

\begin{abstract}
Breeding systems of Melastomataceae in Serra do Japi, Jundiaí, São Paulo, Brazil). The breeding systems of some Melastomataceae species were investigated in populations from forests on the Serra do Japi, southeastern Brazil. This study was based on controlled pollinations and analysis of pollen viability and pollen-tube growth. Among populations of the 13 species studied, seven showed apomictic fruits (Leandra australis, L. melastomoides, L. purpurascens, Miconia latecrenata, M. petropolitana, Ossaea amygdaloides e $O$. confertiflora). Among the six non-apomictic species only one showed selfincompatibility (Miconia pusilliflora), whereas the others showed self-compatibility (Leandra dasytricha, L. regnellii, Tibouchina cerastifolia, T. sellowiana e T. semidecandra). Pollen analysis showed that the apomictic species have significantly lower viability levels than the non-apomictic species.
\end{abstract}

RESUMO - (Sistemas reprodutivos de espécies de Melastomataceae da Serra do Japi, Jundiaí, São Paulo, Brasil). Neste trabalho foram investigados os sistemas reprodutivos de espécies de Melastomataceae ocorrentes em formações florestais da Serra do Japi. Esta investigação foi baseada em polinizações controladas e em análises de viabilidade de pólen e de crescimento de tubo polínico. Entre as populações de 13 espécies estudadas, sete mostraram produção de frutos apomíticos (Leandra australis, L. melastomoides, L. purpurascens, Miconia latecrenata, M. petropolitana, Ossaea amygdaloides e O. confertiflora). Entre as seis espécies não apomíticas, apenas uma apresentou mecanismo de auto-incompatibilidade (Miconia pusilliflora), enquanto que as demais se mostraram auto-compatíveis (Leandra dasytricha, L. regnellii, Tibouchina cerastifolia, T. sellowiana e T. semidecandra). A análise de pólen mostrou que as espécies apomíticas produzem uma proporção de grãos viáveis significativamente menor do que aquelas que não são apomíticas.

Key Words - Plant reproduction, breeding systems, apomixis, Melastomataceae

\section{Introdução}

Melastomataceae é uma família que compreende 4200-4500 espécies e é bem representada em ecossistemas tropicais e subtropicais das Américas, onde são encontradas cerca de 3000 espécies (Renner 1993). As flores destas espécies são geralmente polinizadas por abelhas que coletam o pólen disposto em anteras tubulosas, com forma variável mas quase sempre com deiscência poricida. Estas abelhas abraçam as anteras e efetuam movimentos vibratórios para extrair o pólen das anteras, efetuando polinização vibrátil ("buzz pollination”, Buchmann 1983). Algumas espécies de Melastomataceae, no entanto, apresentam produção de néctar e são polinizadas por

1. Universidade Federal do Paraná, Setor de Ciências Biólogicas, Departamento de Botânica, Caixa Postal 19031, 81531-970, Curitiba, PR, Brasil.

2. Universidade Estadual de Campinas, Instituto de Biologia, Departamento de Botânica, Pós-Graduação em Ecologia.

3. Autor para correspondência: rgolden@bio.ufpr.br ratos (Lumer 1980), moscas (Goldenberg \& Shepherd 1998), morcegos ou beija-flores (Renner 1989).

O sistema reprodutivo predominante na família é o xenógamo (Renner 1989), favorecido pela separação espacial entre o estigma e o pólen nas flores, e pela necessidade de manipulação das anteras por parte de vetores para liberar este pólen. A existência de mecanismos de auto-incompatibilidade genética é relativamente comum nas tribos Rhexieae e Miconieae, e menos característica nas tribos Melastomeae e Microliceae (Goldenberg \& Shepherd 1998).

Trabalhos recentes têm mostrado, com relativa freqüência, a ocorrência de casos de apomixia na família (Renner 1989, Borges 1991, Goldenberg \& Shepherd 1998, Melo et al. 1999, Goldenberg 2000a). A apomixia é um fenômeno pelo qual uma planta é capaz de produzir sementes com embriões viáveis sem que antes houvesse fusão de gametas e formação de zigoto (Nogler 1984, Mogie 1992). A apomixia parece estar relacionada também à poliploidia e hibridações, o que conseqüentemente faz com que a viabilidade do pólen das espécies apomíticas seja quase sempre mais baixa do que nas 
espécies não apomíticas (Grant 1981). Em Melastomataceae, a ocorrência de apomixia parece ser mais freqüente entre espécies da tribo Miconieae (gêneros Miconia, Leandra, Clidemia, entre outros) do que nas demais e aparentemente não tem relação com um tipo de habitat específico (Goldenberg \& Shepherd 1998).

Este trabalho tem como objetivo determinar os sistemas reprodutivos de Melastomataceae que ocorrem na Serra do Japi, uma área rica em espécies desta família, com vegetação de transição entre floresta ombrófila ("Mata Atlântica") e floresta mesófila semidecídua. Estes dados serão comparados com padrões de distribuição de sistemas reprodutivos encontrados em espécies de Melastomataceae de outras áreas, principalmente no que se refere à apomixia.

\section{Material e métodos}

Este trabalho foi conduzido na Reseva Ecológica da Serra do Japi, mantida pela prefeitura do município de Jundiaí, SP. A Serra do Japi situa-se a cerca de $46^{\circ} 53^{\prime}-47^{\circ} 05^{\prime}$ W e $23^{\circ} 13^{\prime}-23^{\circ} 19^{\prime} \mathrm{S}$ e é caracterizada por altitudes que variam entre cerca de 700 e $1300 \mathrm{~m}$ acima do nível do mar. Suas declividades são maiores que $15 \%$, tendo a maior parte de suas cristas angulosas, com vertentes côncavo retilíneas muito ravinadas e vales fechados (Santoro \& Machado 1992). A precipitação anual média é de $1501 \mathrm{~mm}$ e a temperatura média anual varia entre 15,7 e $19,2{ }^{\circ} \mathrm{C}$, de acordo com a altitude (Pinto 1992). A vegetação é formada por floresta mesófila semidecídua de altitude, com transição para floresta mesófila semidecídua e com a presença de elementos provenientes de Mata Atlântica (Leitão-Filho 1992, Rodrigues \& Shepherd 1992). As espécies estudadas encontram-se todas entre 800 e $1100 \mathrm{~m}$ de altitude, em encostas íngremes, sobre latossolo vermelho-amarelo ou litossolo.

Um total de 13 espécies de Melastomataceae foi estudado, sendo cinco espécies de Leandra, três de Miconia e duas de Ossaea, da tribo Miconieae, e três espécies de Tibouchina, da tribo Melastomeae (classificação em tribos segundo Renner 1993). Os sistemas reprodutivos foram investigados através de polinizações controladas, análise de viabilidade de pólen e de crescimento de tubo polínico.

As polinizações controladas foram efetuadas com base em cinco tratamentos (Goldenberg \& Shepherd 1998): testemunha (flores apenas marcadas, não ensacadas); apomixia (botões com estiletes cortados, inflorescências ensacadas); polinização cruzada (flores polinizadas com pólen de outros indivíduos, inflorescências ensacadas), auto-polinização (flores polinizadas com pólen proveniente do próprio indivíduo, inflorescências ensacadas), e auto-polinização espontânea (inflorescências apenas ensacadas). Os tratamentos de polinização cruzada e auto-polinização não foram efetuados nas espécies onde já havia sido detectada a ocorrência de apomixia. A remoção do pólen das anteras foi efetuada cortando-se o ápice das mesmas e pressionando-as na base.
Em Tibouchina sellowiana (Cham.) Cogn. a polinização cruzada foi feita separadamente, com flores de um e dois dias visando investigar uma possível relação entre a viabilidade floral e a mudança de cor nas pétalas. Sempre que possível o mesmo indivíduo recebeu todos os tratamentos, e ao menos 3 indivíduos foram utilizados por espécie, à exceção de Miconia petropolitana Cogn. (apenas 1 indivíduo encontrado na região). O desenvolvimento dos frutos foi acompanhado até 1-2 meses após as polinizações. A partir do número de flores utilizadas e do número de frutos formados por tratamento foi calculada a percentagem de frutos formados.

A análise da viabilidade de pólen foi feita a partir de material fixado (FAA 50\%), colorido com carmim-acético e observado em microscópio óptico. O material foi coletado a partir de 10 indivíduos, à exceção de $M$. petropolitana (1 indivíduo), Leandra australis (Cham.) Cogn., Ossaea amygdaloides (DC.) Triana e O. confertiflora (DC.) Triana (3 indivíduos cada), das quais não foram encontradas mais plantas na região. Para cada indivíduo foram analisadas 3 flores, sendo contados 100 grãos por flor, à exceção das duas espécies de Ossaea, para as quais não foi encontrado pólen fértil e a quantidade de grãos encontrados por flor, mesmo os mal-formados, não atingiu este número. Da proporção entre grãos viáveis e não viáveis - não coloridos ou mal-formados - foi calculada a percentagem de grãos viáveis em relação à soma para cada indivíduo e para cada espécie.

As diferenças de viabilidade polínica entre as espécies foram testadas por análise de variância (ANOVA). A homogeneidade de variância foi testada pelo teste de Bartlett, e a normalidade pelo teste de Kolmogorov-Smirnov (Zar 1996). Para avaliar quais das espécies apresentaram diferença de viabilidade dentro dos grupos foram feitas comparações múltiplas, a posteriori, usando o teste de Scheffé (Zar 1996). Tendo em vista a distribuição binomial dos dados, estes foram transformados por arcoseno da raiz da proporção (Zar 1996). Miconia petropolitana não entrou nas análises por apresentar uma única medida.

A análise do desenvolvimento dos tubos polínicos foi feita a partir de material fixado (FAA 50\%). Após tratamento (em $\mathrm{NaOH} 6 \mathrm{M}$, a $60^{\circ} \mathrm{C}$ por $40 \mathrm{~min}$.), este material foi corado com azul de anilina e observado em microscopia de fluorescência (Martin 1959).

Os materiais-testemunha das espécies estudadas estão depositados no herbário UEC da Universidade Estadual de Campinas, com a seguinte numeração (todas coletas de R. Goldenberg): 149, L. australis (Cham.) Cogn.; 114 e 148, L. dasytricha (A. Gray) Cogn.; 143, L. melastomoides Raddi; 103 e 401, L. purpurascens (DC.) Cogn.; 104, L. regnellii (DC.) Cogn.; 158 e 395, M. latecrenata (DC.) Naudin; 94 e 403, M. petropolitana Cogn.; 37 e 38, M. pusilliflora (DC.) Naudin; 157, O. amygdaloides (DC.) Triana; 402, O. confertiflora (DC.) Triana; 39 e 140, T. cerastifolia (Naudin) Cogn.; 155, T. sellowiana (Cham.) Cogn. e 139, T. semidecandra (DC.) Cogn.

\section{Resultados}

As populações de sete entre as 13 espécies de Melastomataceae estudadas que ocorrem na Serra do Japi apresentaram frutos e sementes formados a 
partir de apomixia: Leandra australis, L. melastomoides, L. purpurascens, Miconia latecrenata, M. petropolitana, Ossaea amygdaloides e $O$. confertiflora (tabela 1).

As populações das demais espécies estudadas não apresentaram frutos e sementes formados a partir de apomixia (tabela 1) ou apresentaram uma proporção extremamente baixa de frutos neste tratamento, como é o caso de L. regnellii, onde apenas 1 flor entre 75 testadas produziu fruto. Das seis espécies não apomíticas, somente $M$. pusilliflora apresentou auto-incompatibilidade, produzindo apenas dois frutos entre 491 flores testadas no tratamento de auto-polinização.

Cinco espécies apresentaram populações não apomíticas e auto-compatíveis: Leandra dasytricha, L. regnellii, Tibouchina cerastifolia, $T$. sellowiana e $T$. semidecandra. Destas, apenas duas espécies apresentaram frutos formados a partir de autopolinização espontânea (tabela 1): Tibouchina sellowiana teve apenas 3,4\% e L. regnellii $10 \%$ de frutos formados neste tratamento.

Tibouchina sellowiana possui flores com pétalas brancas ao abrir e que mudam de cor com o passar do tempo, até atingir uma coloração purpúrea no dia seguinte. Os verticilos nestas flores permanecem intactos, a partir da antese, por dois a três dias, quando inicia a queda das pétalas, estames e estiletes. A proporção de frutos formados em polinizações efetuadas em flores de $2^{\circ}$ dia foi inferior àquelas efetuadas em flores do $1^{\circ}$ dia ( $35 \%$ contra $\left.80 \%\right)$.

Em todas as espécies estudadas - inclusive naquelas com estiletes mais compridos, como $T$. semidecandra e T. sellowiana - os tubos polínicos atingiram os óvulos entre 24 e 48 h após a polinização. A análise dos tubos polínicos provenientes de flores auto-polinizadas de M. pusilliflora mostrou que há uma interrupção no crescimento dos mesmos no terço intermediário dos estiletes.

As análises de viabilidade de pólen (tabela 1; figura 1) mostraram que, em geral, as espécies apomíticas produzem uma proporção de grãos viáveis bem menor do que aquelas que não são apomíticas. Entre as espécies apomíticas os valores médios de viabilidade de pólen variaram entre $0 \mathrm{e}$ $17,1 \%$, à exceção de M. petropolitana, com $60,4 \%$ de grãos viáveis. Os dados sobre esta espécie basearamse em apenas um indivíduo encontrado em estágio

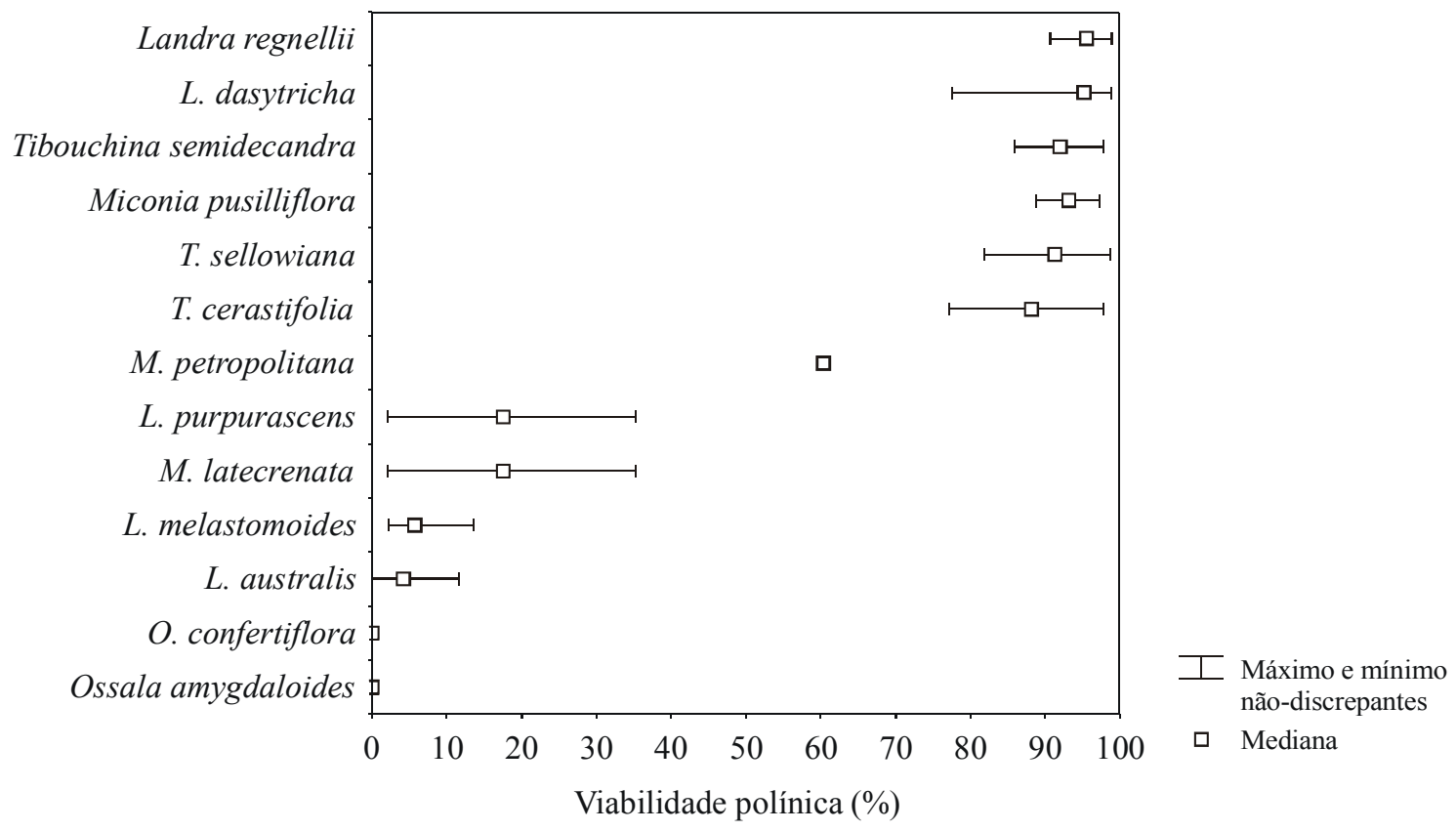

Figura 1. Variação na viabilidade de pólen entre indivíduos das espécies de Melastomataceae estudadas na Serra do Japi. As espécies estão ordenadas em ordem decrescente de valores médios de viabilidade de pólen. As sete primeiras espécies são apomíticas. 
Tabela 1. Polinizações controladas, sistemas reprodutivos e viabilidade de pólen. Polinizações controladas em \% de frutos formados, com o número de flores testadas por tratamento entre parenteses; viabilidade de pólen em percentagem mais ou menos desvio padrão da média. nPC: número de indivíduos utilizados nas polinizações controladas; nVP: número de indivíduos utilizados para análise de viabilidade de pólen; AP: apomixia; AC: auto-compatibilidade; AI: auto-incompatibilidade.

\begin{tabular}{|c|c|c|c|c|c|c|c|c|c|}
\hline \multirow[t]{2}{*}{ Espécies } & \multirow[b]{2}{*}{$\mathrm{nPC}$} & \multicolumn{5}{|c|}{ Polinizações Controladas } & \multirow[t]{2}{*}{$\begin{array}{l}\text { Sistema } \\
\text { Reprod. }\end{array}$} & \multicolumn{2}{|c|}{ Viabilidade de Pólen } \\
\hline & & Apomixia & $\begin{array}{c}\text { Polinização } \\
\text { Cruzada }\end{array}$ & $\begin{array}{c}\text { Auto- } \\
\text { Polinização }\end{array}$ & $\begin{array}{c}\text { Auto- } \\
\text { Espontânea }\end{array}$ & Controle & & \% Grãos Viáveis & $\mathrm{nVP}$ \\
\hline \multicolumn{10}{|l|}{ - Apomíticas } \\
\hline Leandra australis (Cham.) Cogn. & 3 & $90,0(18)$ & - & - & $90,9(22)$ & $93,5(31)$ & $\mathrm{AP}$ & $4,9 \pm 4,0 \mathrm{a}^{* * *}$ & 3 \\
\hline L. melastomoides Raddi & 3 & $95,5(44)$ & - & - & $87,5(24)$ & $82,4(34)$ & $\mathrm{AP}$ & $6,9 \pm 4,5 \mathrm{ab}$ & 10 \\
\hline L. purpurascens (DC.) Cogn. & 3 & $19,4(31)$ & - & - & $46,7(30)$ & $37,5(32)$ & $\mathrm{AP}$ & $17,1 \pm 9,5 b$ & 10 \\
\hline Miconia petropolitana Cogn. & 1 & $88,2(51)$ & - & - & $90,9(11)$ & $87,1(101)$ & $\mathrm{AP}$ & 60,4 & 1 \\
\hline M. latecrenata (DC.) Naudin & 3 & $71,0(69)$ & $78,0(82)$ & - & $73,8(42)$ & $87,1(178)$ & $\mathrm{AP}$ & $9,6 \pm 5,4 b$ & 10 \\
\hline Ossaea amygdaloides (DC.) Triana & 3 & $88,5(26)$ & - & - & $88,2(17)$ & $86,2(29)$ & $\mathrm{AP}$ & $0 \mathrm{a}$ & 3 \\
\hline $\begin{array}{l}\text { O. confertiflora (DC.) Triana. } \\
\text { - Sexuadas }\end{array}$ & 3 & $65,5(29)$ & - & - & $69,0(29)$ & $57,7(26)$ & $\mathrm{AP}$ & $0 \mathrm{a}$ & 3 \\
\hline Leandra dasytricha (A. Gray) Cogn. & 3 & $0(35)$ & $55,2(29)$ & $62,5(24)$ & $0(30)$ & $45,7(35)$ & $\mathrm{AC}$ & $92,3 \pm 7,1 \mathrm{c}$ & 10 \\
\hline L. regnellii (Triana) Cogn. & 4 & $1,3(75)$ & $86,7(21)$ & $77,8(24)$ & $10,0(30)$ & $68,8(48)$ & $\mathrm{AC}$ & $94,5 \pm 4,4 \mathrm{c}$ & 10 \\
\hline Miconia pusilliflora (DC.) Naudin & 3 & $0(242)$ & $20,2(511)$ & $0,4(491)$ & $0(387)$ & $15,0(512)$ & $\mathrm{AI}$ & $90,8 \pm 9,1 \mathrm{c}$ & 10 \\
\hline Tibouchina cerastifolia (Naudin) Cogn. & 4 & $0(16)$ & $56,3(16)$ & $71,4(21)$ & $0(16)$ & $59,0(39)$ & $\mathrm{AC}$ & $86,3 \pm 9,3 \mathrm{c}$ & 10 \\
\hline T. sellowiana (Cham.) Cogn. & 3 & $0(16)$ & $\begin{array}{l}80,0(20)^{*} \\
35,0(20)^{* *}\end{array}$ & $71,4(21)$ & $3,4(29)$ & $76,5(34)$ & $\mathrm{AC}$ & $89,6 \pm 9,6 \mathrm{c}$ & 10 \\
\hline T. semidecandra (DC.) Cogn. & 3 & $0(15)$ & $62,5(16)$ & $75,0(16)$ & $0(16)$ & $58,3(24)$ & $\mathrm{AC}$ & $92,2 \pm 3,6 \mathrm{c}$ & 10 \\
\hline
\end{tabular}

* Flores abertas no primeiro dia;

** Flores de segundo dia

*** Valores seguidos por letras iguais não diferem significativamente entre si.

reprodutivo, onde a distinção entre grãos de pólen viáveis e não viáveis não foi muito clara, pois foram encontrados muitos grãos em estados intermediários entre aqueles completamente vazios e os bem formados. Os valores das espécies com baixa viabilidade foram distintos entre si (ANOVA: $\mathrm{F}_{5,38}=$ 9,34, $\mathrm{P}=0,0000)$ : $L$. australis, $O$. amygdaloides e $O$. confertiflora difereriram de $L$. purpurascens e $M$. latecrenata, enquanto que L. melastomoides não diferiu das demais. Entre as espécies não apomíticas, os valores médios de viabilidade de pólen variaram entre 86,3 e $94,5 \%$, não havendo diferença significativa entre eles (ANOVA: $\mathrm{F}_{5,54}=1,40, \mathrm{P}=$ $0,2389)$.

\section{Discussão}

A ocorrência de sete espécies de Melastomataceae apomíticas entre as 13 estudadas na Serra do Japi mostra que a apomixia é comum entre as espécies da família nesta área. Entre as espécies apomíticas, a maioria pode ser considerada como fazendo parte dos "elementos provenientes de Mata Atlântica" citados por Leitão-Filho (1992), visto que são mais comuns em florestas ao longo das serras próximas à costa. Melastomataceae apomíticas já foram encontradas em formações florestais amazônicas (Renner 1989) e atlânticas (Melo \& Machado 1996, Melo et al. 1999), florestas nebulares venezuelanas (Sobrevila \& Arroyo 1982), florestas estacionais do sudeste do Brasil (Borges 1991), formações alagadas (Ramirez \& Brito 1990) e cerrados (Goldenberg \& Shepherd 1998). A ocorrência de apomixia aparentemente não está relacionada a adaptações a determinadas condições ambientais ou ecológicas, estando mais claramente ligada à expressão ou consequência de outros processos, tais quais hibridação e poliploidia (Goldenberg 2000a). Esta ocorrência também está relacionada a alguns grupos de plantas que têm um conjunto de "pré-adaptações" que permitem o desenvolvimento de embriões apomíticos, tais como Asteraceae, Rosaceae, Poaceae (Mogie 1992) e Melastomataceae tribo Miconieae.

As sete espécies apomíticas encontradas neste trabalho pertencem aos gêneros Leandra, Miconia e Ossaea, todos por sua vez pertencentes à tribo Miconieae. A grande maioria das espécies apomíticas encontradas até o momento em Melastomataceae pertence a esta tribo (Goldenberg \& Shepherd 1998). 
Entre as seis espécies não apomíticas, três pertencem ao gênero Tibouchina, da tribo Melastomaeae (Renner 1993), e 3 a gêneros de Miconieae (Leandra e Miconia). Trabalhos com espécies de vários gêneros de Melastomeae, inclusive Tibouchina, mostram que, em sua maioria, estas também não apresentam apomixia e mecanismos de auto-incompatibilidade (Goldenberg \& Shepherd 1998).

A ocorrência do único caso de autoincompatibilidade, justamente em um representante de Miconieae (M. pusilliflora), também segue um padrão encontrado por Goldenberg \& Shepherd (1998). A ocorrência de casos de apomixia é maior em grupos onde é também maior a freqüência de casos de auto-incompatibilidade, embora não haja uma explicação para este fato (Richards 1986). A interrupção no crescimento dos tubos polínicos no terço intermediário dos estiletes, como a encontrada em M. pusilliflora, é característica de sistemas gametofíticos de incompatibilidade (Gibbs 1990).

A ocorrência de uma proporção muito pequena de frutos formados nos tratamentos de agamospermia em L. regnellii (1 entre 75), de auto-polinização em M. pusilliflora (2 entre 491) e de auto-polinização espontânea em T. sellowiana (1 entre 29) pode estar indicando níveis muito baixos de ocorrência dos fenômenos relacionados aos tratamentos ou pode ser conseqüência de erro na condução do experimento.

Para as espécies aqui estudadas, aparentemente, não é válida a suposição de Goldenberg (2000a) de que as espécies apomíticas têm, geralmente, distribuição mais ampla do que as não apomíticas. No gênero Miconia, a única espécie não apomítica (M. pusilliflora) ocorre nas regiões Sudeste e Sul do Brasil, Paraguai e Argentina, enquanto que, entre as espécies apomíticas, $M$. petropolitana ocorre no Sudeste e Sul do Brasil e Argentina, e M. latecrenata no Nordeste, Sudeste e Sul do Brasil (Goldenberg 2000b). Em Leandra, três espécies ocorrem apenas nas regiões Sudeste e Sul do Brasil: L. australis (apomítica), L. dasytricha e L. regnellii (ambas não apomíticas). As demais espécies (ambas apomíticas) são $L$. purpurascens, que ocorre nas regiões Nordeste, Sudeste e Sul do Brasil, e L. melastomoides (incluindo um provável sinônimo, L. scabra Cogn.), que ocorre no Centro-Oeste, Sudeste e Sul do Brasil (Wurdack 1962).

Apesar de ter sido encontrada diferença entre os níveis de viabilidade de pólen das espécies apomíticas e aqueles das espécies não apomíticas, os dados obtidos não permitem estabeler um nível indicativo da ocorrência de apomixia, ao menos em nível individual. Para o nível de população ou de espécie é possível supor que médias acima de $85 \%$ de grãos viáveis indicam populações não apomíticas, enquanto que médias inferiores a $20 \%$ indicam populações apomíticas. Valores intermediários a estes poderiam incluir indivíduos apomíticos com viabilidade de pólen alta, como é o caso do único indivíduo de $M$. petropolitana estudado, ou indivíduos não apomíticos com viabilidade mais baixa, como a encontrada em alguns indivíduos de $L$. dasytricha e T. cerastifolia (figura 1).

Quanto à relação entre a presença de apomixia e poliploidia, existem publicadas contagens de números cromossômicos apenas para duas das espécies estudadas. A contagem de $2 n=54$ e 60 obtida para $T$. semidecandra refere-se, verdadeiramente, a $T$. urvilleana (Favarger 1967 apud Solt \& Wurdack 1980, Almeda \& Chuang 1992) e portanto não será considerada. Para T. sebastianopolitana (Raddi) Cogn., que provavelmente é conspecífica a $T$. cerastifolia, foi obtido $\mathrm{n}=9$ (Solt \& Wurdack 1980). Segundo Almeda (1997), o número básico de cromossomos em Tibouchina é n $=9$ e, portanto, ao menos para $T$. cerastifolia confirma-se a relação entre a ausência de poliploidia e a ausência de apomixia (Goldenberg \& Shepherd 1998).

A investigação sobre o sistema reprodutivo de T. sellowiana mostrou que as polinizações em flores de $2^{\circ}$ dia tem menor sucesso do que aquelas em flores de $1^{\circ}$ dia. Isso ocorre provavelmente porque os tubos polínicos levam entre 24 e $48 \mathrm{~h}$ para atingir os óvulos e é neste intervalo de tempo que os estiletes começam a cair. Em alguns casos não haveria tempo, portanto, para que os tubos polínicos tivessem seu desenvolvimento completado em flores polinizadas no $2^{\circ}$ dia. É interessante notar que as abelhas que visitam as flores de T. sellowiana parecem abordar apenas flores de $1^{\circ}$ dia, e nunca flores de $2^{\circ}$ dia. É possível que as abelhas reconheçam que a probabilidade de se encontrar o recurso (pólen) seja menor em flores do $2^{\circ}$ dia, e que a mudança na coloração das pétalas sirva de sinal para as abelhas.

Ainda com relação à atividade de abelhas, é interessante notar que estas não foram vistas visitando as espécies apomíticas, enquanto que visitas às espécies não apomíticas foram freqüentemente observadas. Esta ausência de visitas 
por parte de abelhas (Goldenberg \& Shepherd 1998) ou sua ocorrência apenas esporádica (Melo et al. 1999) em espécies apomíticas já havia sido notada anteriormente.

Neste trabalho foi efetuado o primeiro registro de ocorrência de apomixia para o gênero Ossaea. Para o gênero Leandra, a ocorrência de espécies não apomíticas e auto-compatíveis ( $L$. dasytricha e $L$. regnellii) foi registrada pela primeira vez, visto que as três espécies investigadas até o momento eram apomíticas (Goldenberg \& Shepherd 1998) .

Agradecimentos - Gostaríamos de agradecer a Prefeitura de Jundiaí e à direção da Reserva Ecológica da Serra do Japi pela permissão para a condução dos trabalhos de campo, ao Laboratório de Biossistemática do Depto. de Botânica da UNICAMP e ao Prof. Paulo Eugênio M. de Oliveira pela leitura crítica de versões prévias deste trabalho.

\section{Referências bibliográficas}

ALMEDA, F. 1997. Chromosome numbers and their evolutionary significance in some neotropical and paleotropical Melastomataceae. BioLlania Edicion Especial 6:167-190.

ALMEDA, F. \& CHUANG, T.I. 1992. Chromosome numbers and their systematic significance in some mexican Melastomataceae. Systematic Botany 17:583-593.

BORGES, H.B.N. 1991. Biologia reprodutiva de quatro espécies de Melastomataceae. Dissertação de mestrado, Universidade Estadual de Campinas, Campinas.

BUCHMANN, S.L. 1983. Buzz pollination in Angiosperms. In Handbook of experimental pollination biology (C.E. Jones \& R.J. Little, eds.). Van Nostrand Reinhold, New York, p.73-113.

GIBBS, P. 1990. Self-incompatibility in flowering plants: a neotropical perspective. Revista Brasileira de Botânica 13:125136.

GOLDENBERG, R. \& SHEPHERD, G.J. 1998. Studies on the reproductive biology of Melastomataceae in cerrado vegetation. Plant Systematics and Evolution. 211:1329.

GOLDENBERG, R. 2000a. Apomixia como alternativa à reprodução sexuada em Melastomataceae. In Tópicos Atuais em Botânica (T.B. Cavalcanti \& B.M.T.Walter, eds.). EMBRAPA Recursos Genéticos, Brasília, p.225230.

GOLDENBERG, R. 2000b. O Gênero Miconia Ruiz et Pavon (Melastomataceae): I. Listagens Analíticas; II. Revisão Taxonômica da Seção Hypoxanthus (Rich. ex DC.)
Hook.f. Tese de doutorado, Universidade Estadual de Campinas, Campinas.

GRANT, V. 1981. Plant Speciation. Columbia University Press, New York.

LEITÃO-FILHO, H.L. 1992. A flora arbórea da Serra do Japi. In História natural da Serra do Japi (L.P.C. Morelatto, ed.). Editora da UNICAMP, Campinas, p.40-62.

LUMER, C. 1980. Rodent pollination of Blakea (Melastomataceae) in a Costa Rican cloud Forest. Brittonia 32:512-517.

MARTIN, F.N. 1959. Staining and observing pollen tubes in the style by means of fluorescence. Stain Technology 34:125-128.

MELO, G.F. \& MACHADO, I.C. 1996. Biologia da reprodução de Henriettea succosa DC. (Melastomataceae). Revista Brasileira de Biologia 56:383-389.

MELO, G.F., MACHADO, I.C. \& LUCEÑO, M. 1999. Reprodución de tres especies de Clidemia (Melastomataceae) en Brasil. Revista de Biologia Tropical 47:359-363.

MOGIE, M. 1992. The evolution of asexual reproduction in plants. Chapman \& Hall, London.

NOGLER, G.A. 1984. Gametophytic apomixis. In Embriology of Angiosperms (B.M. Johri, ed.). Springer-Verlag, Berlin, p.475518.

PINTO, H.S. 1992. Clima da Serra do Japi. In História natural da Serra do Japi (L.P.C. Morelatto, ed.). Editora da UNICAMP, Campinas, p.30-38.

RAMIREZ, N. \& BRITO, Y. 1990. Reproductive biology of a tropical palm swamp community in the Venezuelan llanos. American Journal of Botany 77:1260-1271.

RENNER, S.S. 1989. A survey of reproductive biology in Neotropical Melastomataceae and Memecylaceae. Annals of the Missouri Botanical Garden 76:496-518.

RENNER, S.S. 1993. Phylogeny and classification of the Melastomataceae and Memecylaceae. Nordic Journal of Botany 13:519-540.

RICHARDS, A.J. 1986. Plant Breeding Systems. Allen \& Unwin, London.

RODRIGUES, R.R. \& SHEPHERD, G.J. 1992. Análise da variação estrutural e fisionômica da vegetação e características edáficas, num gradiente altitudinal na Serra do Japi. In História natural da Serra do Japi (L.P.C. Morelatto, ed.). Editora da UNICAMP, Campinas, p.64-96.

SANTORO, E. \& MACHADO, D.L. 1992. Elementos geológicos da Serra do Japi. In História natural da Serra do Japi (L.P.C. Morelatto, ed.). Editora da UNICAMP, Campinas, p.24-29.

SOBREVILA, C. \& ARROYO, M.T.K. 1982. Breeding systems in a montane tropical cloud forest in Venezuela. Plant Systematics and Evolution 140:19-37.

SOLT, M.L. \& WURDACK, J.J. 1980. Chromossome number in Melastomataceae. Phytologia 47:199-220.

WURDACK, J.J. 1962. Melastomataceae of Santa Catarina. Sellowia 14:109-217.

ZAR, J.H. 1996. Biostatistical analysis. Prentice-Hall, Londres. 